Free centre-by-metabelian Lie algebras in characteristic 2

Kovács, L. G. and Stöhr, Ralph

2013

MIMS EPrint: 2013.19

Manchester Institute for Mathematical Sciences

School of Mathematics

The University of Manchester

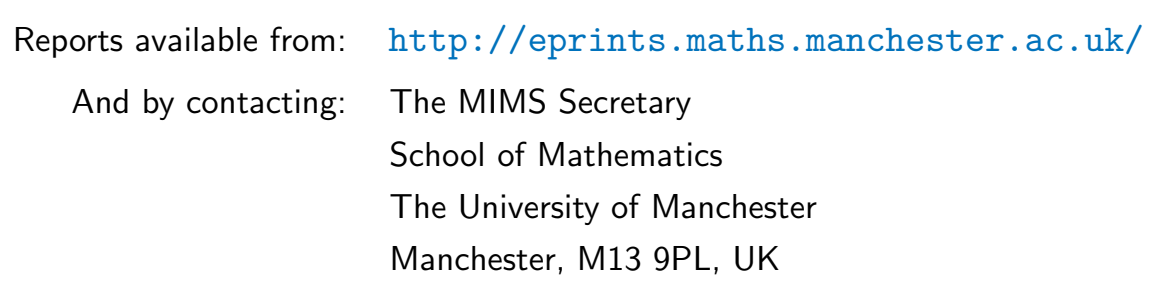

ISSN 1749-9097 


\title{
FREE CENTRE-BY-METABELIAN LIE ALGEBRAS IN CHARACTERISTIC 2
}

\author{
L. G. KOVÁCS AND RALPH STÖHR
}

\begin{abstract}
We study free centre-by-metabelian Lie algebras over a field of characteristic 2. By using homological methods, we determine the dimensions of the fine homogeneous components of the second derived algebra. In conjunction with earlier results by Mansuroğlu and the second author, this leads to a complete description of the additive structure of the second derived ideal in the free centre-by-metabelian Lie ring.
\end{abstract}

\section{INTRODUCTION}

Let $G$ denote the free centre-by-metabelian Lie algebra of finite rank $r>1$ over a commutative ring $K$ with 1 , and let $X$ be a free generating set for $G$. Then $G$ is a central extension of the free metabelian Lie algebra $G / G^{\prime \prime}$ with kernel $G^{\prime \prime}$. Of course, $G / G^{\prime \prime}$ is well-understood: It is a free $K$-module and the simple basic Lie monomials form a basis of it (see, for example, [1]). The second derived ideal $G^{\prime \prime}$ is much more complicated. When $K=\mathbb{Z}$, this ideal contains elements of order 2, and the 2-torsion occurring in even degrees is very different from the 2-torsion in odd degrees. This was discovered by Yu. V. Kuz'min in his pioneering paper [2]. Much later it turned out (see [7], [4]) that some of the details on Lie rings given in [2] required modification. The results in [4] were conclusive in the case where $K$ is a field of characteristic other than 2, and also for the torsion-free part of $G^{\prime \prime}$ when $K=\mathbb{Z}$. Most of the present paper is devoted to calculating the dimensions of the fine homogeneous components of $G^{\prime \prime}$ under the assumption that $K$ is a field of characteristic 2. Combined with the results in [4], these dimensions determine the full additive structure of $G^{\prime \prime}$ in the $K=\mathbb{Z}$ case as well. We give nontrivial elements in each fine homogeneous component such that the component is the direct sum of the cyclic groups generated by these elements. The approach to the problem offered here seems to be new. It is based on ideas developed in [4], but makes essential use of homological methods.

The paper is organized as follows. Notation and some preliminary notions are introduced in Section 2. In Section 3 we record some facts about the homology of modules over polynomial rings. In Section 4 we discuss tensor products and symmetric and exterior squares of such modules, and in the next two Sections 5 and 6 we determine some low-dimensional homology groups with coefficients in such modules. Our main result, giving the dimensions of the fine homogeneous

2010 Mathematics Subject Classification. Primary 17B01, 17 B55. 
components of $G^{\prime \prime}$, is proved in Section 7, and the application to free centre-bymetabelian Lie rings is carried out in the final Section 8.

\section{Preliminaries}

In what follows $K$ denotes a field or the ring of integers, $X=\left\{x_{1}, x_{2}, \ldots, x_{r}\right\}$ is a finite set with $r>1$. The free centre-by-metabelian Lie algebra on $X$ over $K$ is defined as the quotient

$$
G=L /\left[L^{\prime \prime}, L\right]
$$

where $L$ is the (absolutely) free Lie algebra on $X$ over $K$ and $L^{\prime \prime}$ is its second derived ideal. Thus $G$ is a central extension of the free metabelian Lie algebra $G / G^{\prime \prime}=L / L^{\prime \prime}$ with kernel $G^{\prime \prime}=L^{\prime \prime} /\left[L^{\prime \prime}, L\right]$. Let $M=G^{\prime} / G^{\prime \prime}=L^{\prime} / L^{\prime \prime}$. The adjoint representation in $G$ induces on $M$ the structure of a module for the abelian Lie algebra $G / G^{\prime}$, and hence for the universal envelope $U$ of $G / G^{\prime}$. In fact, this universal envelope is $U=K[X]$, the ring of polynomials in $X$. The starting point of our investigation is the isomorphism

$$
G^{\prime \prime} \cong(M \wedge M) \otimes_{U} K
$$

where the exterior square $M \wedge M$ of the module $M$ is considered as a $U$-module with derivation action, i.e. for $u, v \in M$ and $x \in X$ the action is given by $(u \wedge v) x=u x \wedge v+u \wedge v x$, and $K$ is regarded as a trivial $U$-module (see [4, (2.7)]). More generally, tensor products of $U$-modules as well as exterior and symmetric powers will always be regarded as $U$-modules with derivation action. The exterior, tensor and symmetric squares of any $U$-module $A$ fit into the short exact sequence

$$
0 \rightarrow A \wedge A \rightarrow A \otimes A \rightarrow A \circ A \rightarrow 0
$$

where the relevant maps are given by $a \wedge b \mapsto a \otimes b-b \otimes a$ and $a \otimes b \mapsto a \circ b(a, b \in A)$. If $K$ is a field of characteristic 2, then the exterior square is a homomorphic image of the symmetric square, and there is a short exact sequence

$$
0 \rightarrow D(A) \rightarrow A \circ A \rightarrow A \wedge A \rightarrow 0
$$

where the map on the right is the natural projection given by $a \circ b \mapsto a \wedge b$, and $D(A)$ is the kernel of this map. If $\mathcal{A}$ is a $K$-basis of $A$, then $D(A)$ is precisely the span of all squares $a \circ a$ with $a \in \mathcal{A}$. Note that $D(A)$ is a trivial $U$-module. Indeed, for any $x \in X$ we have $(a \circ a) x=a x \circ a+a \circ a x=0$ as we are in characteristic 2 . Note that characteristic 2 is essential here. If the characteristic of $K$ is not 2, the exact sequence (2.3) is not available.

The augmentation map $\varepsilon: U \rightarrow K$ takes every polynomial in $X$ to its constant term. Its kernel is the augmentation ideal $\Delta$, which consists of the polynomials with zero constant term. The corresponding short exact sequence

$$
0 \rightarrow \Delta \rightarrow U \stackrel{\varepsilon}{\rightarrow} K \rightarrow 0
$$

is referred to as the augmentation sequence. We will assume that the set $X$ is ordered by $x_{1}<x_{2}<\cdots<x_{r}$. The module $M$ has a $K$-basis consisting of the left normed Lie products $\left[y_{1}, y_{2}, \ldots, y_{n}\right]$ with $y_{i} \in X, y_{1}>y_{2} \leqslant y_{3} \leqslant \cdots \leqslant y_{n}, n \geqslant 2$, 
and as a $U$-module it is generated by the Lie products $\left[x_{i}, x_{j}\right]$ with $1 \leqslant j<i \leqslant r$. Let $P$ denote the free $U$-module of rank $r$ with free generators $e_{1}, e_{2}, \ldots, e_{r}$. The map $\left[x_{i}, x_{j}\right] \mapsto e_{i} x_{j}-e_{j} x_{i}$ extends to an embedding $\mu: M \rightarrow P$. Moreover, if $\sigma: P \rightarrow \Delta$ is the map determined by $e_{i} \mapsto x_{i}$, then

$$
0 \rightarrow M \stackrel{\mu}{\rightarrow} P \stackrel{\sigma}{\rightarrow} \Delta \rightarrow 0
$$

is an exact sequence of $U$-modules (see [6]). This yields a 4 -term exact sequence

$$
0 \rightarrow M \wedge M \rightarrow P \wedge P \rightarrow \Delta \otimes P \rightarrow \Delta \circ \Delta \rightarrow 0,
$$

where the maps are (from left to right) given by

$$
\begin{array}{lr}
m_{1} \wedge m_{2} \mapsto m_{1} \mu \wedge m_{2} \mu & \left(m_{1}, m_{2} \in M\right) \\
p_{1} \wedge p_{2} \mapsto p_{1} \sigma \otimes p_{2}-p_{2} \sigma \otimes p_{1} & \left(p_{1}, p_{2} \in P\right) \\
\delta \otimes p \mapsto \delta \circ p \sigma & (\delta \in \Delta, p \in P)
\end{array}
$$

with $\mu$ and $\sigma$ as defined above. A proof of the exactness can be found in [4, Proof of Lemma 5.1]. The exact sequence (2.4) will be one of the main tools in our approach to $G^{\prime \prime}$.

The free centre-by-metabelian Lie algebra $G$, the polynomial ring $U=K[X]$, and all the modules considered so far ( $\operatorname{such}$ as $M, P, \Delta$ ), as well as all tensor, exterior or symmetric products of such modules have natural gradings by degree and, moreover, fine gradings by multidegree with respect to the generating set $X$. The multidegree of a Lie monomial $w \in G$ with factors from $X$ is the $r$-tuple $q=\left(q_{1}, q_{2}, \ldots, q_{r}\right)$ where $q_{i}$ is the partial degree of $w$ with respect to $x_{i} \in X$. The partial degrees add up to the total degree of $w$, say $n=q_{1}+\cdots+q_{r}$. We also refer to $q$ as a composition of $n, q \vDash n$, in $r$ parts. By $G_{n}$ we denote the degree $n$ homogeneous component of $G$, that is the $K$-span of all Lie monomials of degree $n$ in $G$, and we write $G_{q}$ for the span of all Lie monomials of multidegree $q$. The latter is the fine homogeneous component of multidegree $q$. A fine homogeneous component $G_{q}$ and the corresponding multidegree $q$ are called multilinear, if all non-zero parts of $q$ are equal to 1 . It is clear that each $G_{n}$ is the direct sum of the fine homogeneous components $G_{q}$ where $q$ runs over all compositions of $n$. It is also plain that if a composition $q^{\prime}$ can be obtained from $q$ by permuting the parts, then $G_{q}$ and $G_{q^{\prime}}$ are isomorphic as $K$-spaces. In particular, any fine homogeneous component $G_{q}$ is isomorphic to $G_{\tilde{q}}$ where $\tilde{q}$ is the unique partition of $n$ that can be obtained from $q$ by permuting the parts. (Recall that a partition of $n$ is a composition $q=\left(q_{1}, q_{2}, \ldots, q_{r}\right) \vDash n$ such that $q_{1} \geqslant q_{2} \geqslant \cdots \geqslant q_{r}$.) We set $G_{q}^{\prime \prime}=G_{q} \cap G^{\prime \prime}$.

The fine homogeneous components of the polynomial ring $U=K[X]$ and of the various $U$-modules considered so far are defined in a natural way, given that each free generator $e_{i}$ of the module $P$ is assigned the same partial degree as the matching $x_{i}$. For example, the element $e_{1} x_{1} x_{2} \in P$ has degree 3 and multidegree $(2,1,0 \ldots, 0)$ and the element $e_{2} x_{1} \otimes e_{3} \in P \otimes P$ has degree 3 and multidegree $(1,1,1,0, \ldots, 0)$. Note that all the maps considered in this paper respect the fine homogeneous structures, in that they map fine homogeneous components into fine 
homogeneous components, albeit sometimes with a shifted multidegree: in this sense, they are morphisms of finely graded $K$-modules. In the case of the polynomial ring $U=K[X]$, all fine homogeneous components are one-dimensional, and $U_{q}$ is spanned by the unique (commutative and associative) monomial of mutidegree $q$ in $U$. We write $\mathcal{U}$ for the set

$$
\mathcal{U}=\left\{y_{1} y_{2} \ldots y_{k} ; y_{i} \in X, y_{1} \leqslant y_{2} \leqslant \cdots \leqslant y_{k}, k \geqslant 0\right\}
$$

of all monomials in $U$, with the convention that 1 is the only monomial of degree zero. Of course, $\mathcal{U}$ is a $K$-basis of $U$, and $\mathcal{U} \backslash\{1\}$ is a $K$-basis of $\Delta$.

It is easily seen that the smallest non-zero homogeneous component of $G^{\prime \prime}$ is $G_{4}^{\prime \prime} \cong G_{2} \wedge G_{2}$. Most of the rest of this paper is devoted to finding the dimensions of $G_{q}^{\prime \prime}$ for $q \vDash n$ with $n \geqslant 5$.

\section{Homology}

In this section we recall some facts about the homology of $U$-modules. For details we refer to $[3$, Chapter VII]. Recall the $U=K[X]$ is the ring of polynomials in $X$ with coefficients in $K$, and that $P$ is a free $U$-module of rank $r$ with free generators $e_{1}, e_{2}, \ldots, e_{r}$. We let $P_{k}$ denote the $k$-th exterior power of $P$. Thus $P_{k}$ is a free $U$-module with free generators $e_{i_{1}} \wedge e_{i_{2}} \wedge \cdots \wedge e_{i_{k}}$ where $1 \leqslant i_{1}<i_{2}<\cdots<i_{k} \leqslant r$, with the convention that $P_{0}=U$ and $P_{k}=0$ for $k>r$. It is well-known that the complex

$$
\mathrm{P}: \quad 0 \rightarrow P_{r} \stackrel{\partial_{r}}{\longrightarrow} P_{r-1} \stackrel{\partial_{r-1}}{\longrightarrow} \cdots \stackrel{\partial_{3}}{\longrightarrow} P_{2} \stackrel{\partial_{2}}{\longrightarrow} P_{1} \stackrel{\partial_{1}}{\longrightarrow} P_{0} \rightarrow K \rightarrow 0
$$

with differentials given by

$$
\partial_{k}: e_{i_{1}} \wedge e_{i_{2}} \wedge \cdots \wedge e_{i_{k}} \mapsto \sum_{j=1}^{k}(-1)^{j+1}\left(e_{i_{1}} \wedge \cdots \wedge \hat{e}_{i_{j}} \wedge \cdots \wedge e_{i_{k}}\right) x_{i_{j}},
$$

where the circumflex indicates that $e_{i_{j}}$ is to be omitted, is a free resolution of the trivial $U$-module $K$. The complex $\mathrm{P}$ is often called the Koszul complex. Of course, if $K$ is a field of characteristic 2 , the factor $(-1)^{k+1}$ becomes redundant in the definition of the differential. The homology groups of $U$ with coefficients in a $U$-module $A$ are defined by

$$
H_{k}(A)=H_{k}\left(\mathrm{P} \otimes_{U} A\right)
$$

As in the previous section, we assign to each free generator $e_{i}$ of $P$ the same partial degree as that of the matching $x_{i}$. Then the free resolution $\mathrm{P}$ becomes a complex of finely graded $U$-modules. For example, the element $\left(e_{2} \wedge e_{3}\right) x_{1}^{2} \in P_{2}$ has degree 4 and multidegree $(2,1,1,0, \ldots, 0)$. Moreover, if $A$ is a finely graded $U$-module, then the tensor product $\mathrm{P} \otimes A$ too becomes a complex of finely graded $U$-modules. This induces a fine homogeneous structure on the homology groups $H_{k}(A)$ : The homogeneous and fine homogeneous components of those homology groups are defined in the obvious way, and we use $\left(H_{k}(A)\right)_{n}$ for $n \geqslant 0$ and $\left(H_{k}(A)\right)_{q}$ for $q \vDash n$ to denote them.

If $A=K$, all differentials in the complex $\mathrm{P} \otimes_{U} K$ are zero maps, and hence the homology groups $H_{k}(K)$ of the trivial module $K$ are free $K$-modules with $K$-bases 
consisting of the elements $\left(e_{i_{1}} \wedge e_{i_{2}} \wedge \cdots \wedge e_{i_{k}}\right) \otimes 1$ with $1 \leqslant i_{1}<i_{2}<\cdots<i_{k} \leqslant r$. This means that the fine homogeneous components of the homology groups are as follows: For a composition $q \vDash n$, we have

$$
\left(H_{k}(K)\right)_{q}= \begin{cases}K, & \text { if } k \leqslant r, n=k \text { and } q \text { is multilinear } \\ 0, & \text { otherwise }\end{cases}
$$

\section{Tensor Products, SyMmetric AND EXTERIOR SQUARES}

Recall that tensor products of $U$-modules as well as exterior and symmetric powers will always be regarded as $U$-modules with derivation action. The following lemma is well-known. It is, in fact, a special case of a very general result on Hopf algebras (see [5, Theorem 1.9.4]). An elementary proof, tailored to the needs of the present paper, can be found in [4].

Lemma 4.1. Let $N$ be an arbitrary $U$-module that is free as a $K$-module with $K$-basis $\mathcal{N}$. Then the tensor product $N \otimes U$ is a free $U$-module and the elements $m \otimes 1$ with $m \in \mathcal{N}$ form a free generating set for $N \otimes U$ as a $U$-module.

In particular, the tensor square $U \otimes U$ is a free $U$-module with free generators $u \otimes 1$ where $u \in \mathcal{U}$. If $K$ is a field of characteristic other than 2 , then the symmetric and exterior squares $U \circ U$ and $U \wedge U$ are also free $U$-modules. Things, however, are different in characteristic 2, where neither $U \circ U$ nor $U \wedge U$ are free as $U$-modules.

As before, let $P$ be a free $U$-module with free generators $e_{1}, \ldots, e_{r}$. Thus $P=\bigoplus_{i} e_{i} U$, and then $P \wedge P \cong \bigoplus_{i}\left(e_{i} U \wedge e_{i} U\right) \oplus \bigoplus_{i<j}\left(e_{i} U \otimes e_{j} U\right)$. Here $e_{i} U \wedge e_{i} U$ is isomorphic to $U \wedge U$ and $e_{i} U \otimes e_{j} U$ is isomorphic to $U \otimes U$, but it must be noted that these isomorphisms do not preserve the fine gradings. They respect them in the sense previously indicated: for example, given a multidegree $q$, the $U$-module isomorphism between $U \wedge U$ and $e_{i} U \wedge e_{i} U$ matches the fine homogeneous component $(U \wedge U)_{q}$ to $\left(e_{i} U \wedge e_{i} U\right)_{q^{\prime}}$ where $q^{\prime}$ agrees with $q$ except that $q_{i}^{\prime}=q_{i}+2$. Similar comments apply to the tensor product $e_{i} U \otimes e_{j} U$ and the symmetric square $e_{i} U \circ e_{i} U$. We record these simple facts for further reference.

Lemma 4.2. Let $P$ be a free $U$-module with free generators $e_{1}, \ldots, e_{r}$.

(i) For $P \wedge P$ there is a direct decomposition

$$
P \wedge P \cong \bigoplus_{i}\left(e_{i} U \wedge e_{i} U\right) \oplus \bigoplus_{i<j}\left(e_{i} U \otimes e_{j} U\right)
$$

Here each of the direct summands $e_{i} U \wedge e_{i} U$ is isomorphic to $U \wedge U$, and each $e_{i} U \otimes e_{j} U$ is a free $U$-module with free generators $e_{i} u \otimes e_{j}(u \in \mathcal{U})$.

(ii) For $P \circ P$ there is a direct decomposition

$$
P \circ P \cong \bigoplus_{i}\left(e_{i} U \circ e_{i} U\right) \oplus \bigoplus_{i<j}\left(e_{i} U \otimes e_{j} U\right) .
$$

Here each of the direct summands $e_{i} U \circ e_{i} U$ is isomorphic to $U \circ U$, and each $e_{i} U \otimes e_{j} U$ is a free $U$-module with free generators $e_{i} u \otimes e_{j}(u \in \mathcal{U})$. 
The lemma implies that the multilinear components of the homology groups of $P \wedge P$ and $P \circ P$ are zero in all positive dimensions. Namely, all free modules, whether graded or not, have zero homology in all positive dimensions. On the other hand, the fine gradings of the $e_{i} U \wedge e_{i} U$ and $e_{i} U \circ e_{i} U$ being as indicated, the $i$ th partial degree involved in any nonzero fine homogeneous component of such a module is at least 2: so these modules have no multilinear components and hence no multilinear homology. This will be very useful later.

Corollary 4.3. If $q$ is multilinear, then $\left(H_{k}(P \wedge P)\right)_{q}=0$ and $\left(H_{k}(P \circ P)\right)_{q}=0$ for all $k \geqslant 1$.

\section{TRIVIALIZATION OF TENSOR, SYMMETRIC AND EXTERIOR SQUARES}

In this section we work out the trivialization of the tensor, symmetric and exterior squares of free $U$-modules and of the augmentation ideal $\Delta$. For an arbitrary $U$-module $A$, the trivialization of $A$ is the zero-dimensional homology group $H_{0}(A)=A \otimes_{U} K=A / A \Delta$. For the tensor square, $H_{0}(A \otimes A)$ is obtained by forming the quotient of the tensor product $A \otimes A$ by the $K$-submodule generated by the elements of the form $(a \otimes b) x$, that is, by the elements

$$
a x \otimes b+b \otimes a x \quad(a, b \in A, x \in X) .
$$

For simplicity, we denote by $a \otimes_{*} b$ the image of the element $a \otimes b$ of $A \otimes A$ in $H_{0}(A \otimes A)$. Similarly, we use $a \circ_{*} b$ and $a \wedge_{*} b$ for images in the trivializations of the symmetric and exterior squares. In particular, in $H_{0}(A \otimes A)$ the relation

$$
a \otimes_{*} b x=-a x \otimes_{*} b
$$

holds for all $a, b \in A, x \in X$, and similar relations hold in the trivializations of the exterior and symmetric squares of $A$. Further, trivialization yields from (2.2) an exact sequence

$$
H_{0}(A \wedge A) \rightarrow H_{0}(A \otimes A) \rightarrow H_{0}(A \circ A) \rightarrow 0
$$

with the first map $a \wedge_{*} b \mapsto a \otimes_{*} b-b \otimes_{*} a$ and the second $a \otimes_{*} b \mapsto a \circ_{*} b$.

By Lemma 4.1, the tensor product $U \otimes U$ is a free $U$-module with free generators $u \otimes 1(u \in \mathcal{U})$. Hence the trivialization $H_{0}(U \otimes U)$ is a free $K$-module with free generators $u \otimes_{*} 1(u \in \mathcal{U})$. In view of (5.1), we have $u \otimes_{*} v=(-1)^{\operatorname{deg} v} u v \otimes_{*} 1$ and $v \otimes_{*} u=(-1)^{\operatorname{deg} u} u v \otimes_{*} 1$ whenever $u, v \in \mathcal{U}$.

When $K$ is a field of characteristic 2, this implies that the first map in the exact sequence (5.2) with $A=U$ is the zero map. Then the second map is an isomorphism, and we may conclude that in this case $H_{0}(U \circ U)$ is a $K$-space with basis $\left\{u \circ_{*} 1 \mid u \in \mathcal{U}\right\}$. Moreover, now the sequence (2.3) is also available: take it with $A=U$. Then there is an exact sequence

$$
H_{0}(D(U)) \rightarrow H_{0}(U \circ U) \rightarrow H_{0}(U \wedge U) \rightarrow 0 .
$$

As $D(A)$ is always a trivial $U$-module, $H_{0}(D(U))=D(U)$. Hence the term on the left has a $K$-basis consisting of the $u \circ_{*} u$ with $u \in \mathcal{U}$. The map on the left takes such a basis element to $u \circ_{*} u=u^{2} \circ_{*} 1 \in H_{0}(U \circ U)$. We may conclude that 
$H_{0}(U \wedge U)$ has a $K$-basis consisting of the elements $u \wedge_{*} 1$ with $u$ ranging through the non-square elements of $\mathcal{U}$.

We summarize our discussion as follows.

Lemma 5.1. If $K$ is a field of characteristic 2 , then

(i) $H_{0}(U \otimes U)$ is a $K$-space with basis consisting of the elements $u \otimes_{*} 1$ where $u \in \mathcal{U}$

(ii) $H_{0}(U \circ U)$ is a $K$-space with basis consisting of the elements $u \circ_{*} 1$ where $u \in \mathcal{U}$,

(iii) $H_{0}(U \wedge U)$ is a $K$-space with basis consisting of the elements $u \wedge_{*} 1$ where $u \in \mathcal{U}$ but $u$ is not a square.

In view of Lemma 4.2, the following is an easy consequence of this lemma.

Corollary 5.2. If $K$ is a field of characteristic 2, then

(i) $H_{0}(P \otimes P)$ is a $K$-space with basis consisting of the elements $e_{i} u \otimes_{*} e_{j}$ where $1 \leqslant i \leqslant j \leqslant r$ and $u \in \mathcal{U}$,

(ii) $H_{0}(P \circ P)$ is a $K$-space with basis consisting of the elements $e_{i} u \circ_{*} e_{j}$ where $1 \leqslant i \leqslant j \leqslant r$ and $u \in \mathcal{U}$,

(iii) $H_{0}(P \wedge P)$ is a $K$-space with basis consisting of the elements $e_{i} u \wedge_{*} e_{j}$ where $1 \leqslant i<j \leqslant r$ and $u \in \mathcal{U}$, and the elements $e_{i} u \wedge_{*} e_{i}$ where $1 \leqslant i \leqslant r$ and $u \in \mathcal{U}$ such that $u$ is not a square.

Next we examine the tensor, symmetric and exterior squares of the augmentation ideal $\Delta$. The result is very similar to Lemma 5.1 .

Lemma 5.3. If $K$ is a field of characteristic 2, then

(i) $H_{0}(\Delta \otimes \Delta)$ is a $K$-space with basis consisting of the elements $x_{i} \otimes_{*} x_{j}$ where $1 \leqslant i, j \leqslant r$, together with the elements $y_{1} \otimes_{*}\left(y_{2} \cdots y_{n}\right)$ where $y_{i} \in X$ such that $y_{1} \leqslant y_{2} \leqslant \cdots \leqslant y_{n}$ and $n \geqslant 3$,

(ii) $H_{0}(\Delta \circ \Delta)$ is a $K$-space with basis consisting of the elements $y_{1} \circ_{*}\left(y_{2} \cdots y_{n}\right)$ where $y_{i} \in X$ such that $y_{1} \leqslant y_{2} \leqslant \cdots \leqslant y_{n}$ and $n \geqslant 2$,

(iii) $H_{0}(\Delta \wedge \Delta)$ is a $K$-space with basis consisting of the elements $y_{1} \wedge_{*}\left(y_{2} \cdots y_{n}\right)$ where $y_{i} \in X$ such that $y_{1} \leqslant y_{2} \leqslant \cdots \leqslant y_{n}$ and $n \geqslant 2$, but $y_{1} y_{2} \cdots y_{n}$ is not a square.

Proof. The tensor square $\Delta \otimes \Delta$ has a $K$-basis consisting of all elements $u \otimes v$ with $u, v \in \mathcal{U} \backslash\{1\}$. Since the elements in $\Delta \otimes \Delta$ have degree at least 2 and the elements in $(\Delta \otimes \Delta) \Delta$ have degree at least 3 , we have $\left(H_{0}(\Delta \otimes \Delta)\right)_{2}=(\Delta \otimes \Delta)_{2}$. This proves the lemma for degree 2. For a fixed $n \geqslant 3$, in view of the relations (5.1), $\left(H_{0}(\Delta \otimes \Delta)\right)_{n}$ is spanned by the elements $y_{1} \otimes_{*}\left(y_{2} \cdots y_{n}\right)$ where $y_{i} \in X$ such that $y_{1} \leqslant y_{2} \leqslant \cdots \leqslant y_{n}$. Consider the embedding $\Delta \otimes \Delta \rightarrow \Delta \otimes U$. Trivializing yields a homomorphism $H_{0}(\Delta \otimes \Delta) \rightarrow H_{0}(\Delta \otimes U)$. By Lemma 4.1, $H_{0}(\Delta \otimes U)$ has a $K$-basis consisting of the elements $u \otimes_{*} 1$ where $u \in \mathcal{U} \backslash\{1\}$. The image of $y_{1} \otimes_{*}\left(y_{2} \cdots y_{n}\right)$ in $H_{0}(\Delta \otimes U)$ is $\left(y_{1} y_{2} \cdots y_{n}\right) \otimes_{*}$. It follows that the spanning set given above for $\left(H_{0}(\Delta \otimes \Delta)\right)_{n}$ is linearly independent. This proves part (i) of the lemma. For the 
proof of (ii), consider the exact sequence (5.2) with $A=\Delta$, that is,

$$
H_{0}(\Delta \wedge \Delta) \rightarrow H_{0}(\Delta \otimes \Delta) \rightarrow H_{0}(\Delta \circ \Delta) \rightarrow 0 .
$$

It is not hard to see that the image of the left map is spanned by the elements $x_{i} \otimes_{*} x_{j}+x_{j} \otimes_{*} x_{i}$ where $1 \leqslant i, j \leqslant r$. Indeed, the left map in (5.3) takes an element $u \wedge_{*} v \in H_{0}(\Delta \wedge \Delta)$ with $u, v \in \mathcal{U} \backslash\{1\}$ to $u \otimes_{*} v+v \otimes_{*} u$. But if $\operatorname{deg} u+\operatorname{deg} v \geqslant 3$, the relation (5.1) implies that $u \otimes_{*} v=v \otimes_{*} u$; as we are in characteristic 2 , this means that $u \otimes_{*} v+v \otimes_{*} u=0$. We are left with the elements $x_{i} \otimes_{*} x_{j}+x_{j} \otimes_{*} x_{i}$ where $1 \leqslant i, j \leqslant r$. Now (ii) follows from the exactness of the displayed sequence. A similar argument, using the short exact sequence (2.3) with $A=\Delta$, proves (iii).

In view of the explicit bases described here, it is straightforward to count the dimensions of the fine homogeneous components of these homology groups. We shall use the results of two such counts, the first from Lemma 5.3(ii) and the second from Corollary 5.2(iii).

Corollary 5.4. Let $K$ be a field of characteristic 2 .

(i) If $q \vDash n \geqslant 2$, then $\operatorname{dim}\left(H_{0}(\Delta \circ \Delta)\right)_{q}=1$.

(ii) If $q \vDash n \geqslant 2$ with $k$ denoting the number of positive parts of $q$ and $m$ the number of parts that are equal to 1 , then

$$
\operatorname{dim}\left(H_{0}(P \wedge P)\right)_{q}= \begin{cases}\left(\begin{array}{l}
k \\
2
\end{array}\right), & \text { if all parts of } q \text { are even } \\
\left(\begin{array}{l}
k \\
2
\end{array}\right)+k-m, & \text { otherwise. }\end{cases}
$$

6. Low Dimensional HOMOLOGY OF THE SYMMETRIC SQUARE $\Delta \circ \Delta$

In this section we assume that $K$ is a field of characteristic 2. By tensoring the augmentation sequence with $\Delta$, and combining the result with the augmentation sequence itself, we obtain a 4 -term exact sequence

$$
0 \rightarrow \Delta \otimes \Delta \rightarrow \Delta \otimes U \rightarrow U \rightarrow K \rightarrow 0
$$

The two modules in the middle are free, and hence, for all $k \geqslant 1$, there is an isomorphism

$$
H_{k}(\Delta \otimes \Delta) \cong H_{k+2}(K)
$$

Consider the long exact homology sequence stemming from the short exact sequence (2.2) with $A=\Delta$. Here is a part of this sequence:

$$
\begin{aligned}
\cdots \rightarrow H_{2}(\Delta \otimes \Delta) \rightarrow & H_{2}(\Delta \circ \Delta) \rightarrow H_{1}(\Delta \wedge \Delta) \rightarrow H_{1}(\Delta \otimes \Delta) \rightarrow \\
& \rightarrow H_{1}(\Delta \circ \Delta) \rightarrow H_{0}(\Delta \wedge \Delta) \rightarrow H_{0}(\Delta \otimes \Delta) \rightarrow \cdots
\end{aligned}
$$

From (6.1) and (3.1) we see that most fine homogeneous components of $H_{2}(\Delta \otimes \Delta)$ and $H_{1}(\Delta \otimes \Delta)$ vanish, the exceptions occurring in degrees 4 and 3 , respectively. Similarly, a step in the proof of Lemma 5.2(ii) showed that $H_{0}(\Delta \wedge \Delta) \rightarrow H_{0}(\Delta \otimes \Delta)$ 
is the zero map except in degree 2. Thus for $q \vDash n \geqslant 5$, the exactness of (6.2) yields that

$$
\left(H_{2}(\Delta \circ \Delta)\right)_{q} \cong\left(H_{1}(\Delta \wedge \Delta)\right)_{q} \text { and } \quad\left(H_{1}(\Delta \circ \Delta)\right)_{q} \cong\left(H_{0}(\Delta \wedge \Delta)\right)_{q} .
$$

In particular, as we know the fine homogeneous structure of $H_{0}(\Delta \wedge \Delta)$ from Lemma 5.2 (iii), the second part of (6.2) gives the following result.

Lemma 6.1. If $K$ is a field of characteristic 2 and $q \vDash n$ with $n \geqslant 5$, then

$$
\operatorname{dim}\left(H_{1}(\Delta \circ \Delta)\right)_{q}= \begin{cases}0, & \text { if all parts of } q \text { are even } \\ 1, & \text { otherwise. }\end{cases}
$$

Now consider the long exact homology sequence stemming from the short exact sequence (2.3) with $A=\Delta$ :

$$
\begin{aligned}
\cdots \rightarrow H_{1}(D(\Delta)) \rightarrow & H_{1}(\Delta \circ \Delta) \rightarrow H_{1}(\Delta \wedge \Delta) \rightarrow \\
& \rightarrow H_{0}(D(\Delta)) \rightarrow H_{0}(\Delta \circ \Delta) \rightarrow H_{0}(\Delta \wedge \Delta) \rightarrow 0 .
\end{aligned}
$$

By its very nature, $D(\Delta)$ has no multilinear homogeneous components. Hence $\left(H_{k}(D(\Delta))\right)_{q}=0$ for all multilinear multidegrees $q$ and all $k \geqslant 0$. Then the exactness of (6.4) implies that for all multilinear compositions $q \vDash n$ with $n \geqslant 5$ and all $k \geqslant 0$ there are isomorphisms

$$
\left(H_{k}(\Delta \circ \Delta)\right)_{q} \cong\left(H_{k}(\Delta \wedge \Delta)\right)_{q} .
$$

By Lemma 5.2(iii), $\operatorname{dim}\left(H_{k}(\Delta \wedge \Delta)\right)_{q}=1$ for all multilinear compositions $q$ of total degree $n \geqslant 5$, and by combining this with (6.3) and (6.5) we obtain the final result of this section:

Lemma 6.2. If $K$ is a field of characteristic 2, then, for all multilinear compositions $q \vDash n$ with $n \geqslant 5$,

$$
\operatorname{dim}\left(H_{2}(\Delta \circ \Delta)\right)_{q}=\operatorname{dim}\left(H_{1}(\Delta \circ \Delta)\right)_{q}=1 .
$$

\section{Free Centre-By-metabelian Lie algebras in Characteristic 2}

In this section we work out the dimensions of the fine homogeneous components $G_{q}^{\prime \prime}$ for $q \vDash n$ with $n \geqslant 5$, where $G$ is the free centre-by metabelien Lie algebra of rank $r$ over a field $K$ of characteristic 2. In view of the isomorphism (2.1) we can work with the tensor product $(M \wedge M) \otimes_{U} K=H_{0}(M \wedge M)$. Consider the 4-term exact sequence (2.4). Let $W$ denote the kernel of the map $\Delta \otimes P \rightarrow \Delta \circ \Delta$. Then (2.4) can be broken up into two short exact sequences, namely

$$
0 \rightarrow M \wedge M \rightarrow P \wedge P \rightarrow W \rightarrow 0
$$

and

$$
0 \rightarrow W \rightarrow \Delta \otimes P \rightarrow \Delta \circ \Delta \rightarrow 0 .
$$

We first use (7.2) to work out the dimensions of the fine homogeneous components of $H_{0}(W)$ and $H_{1}(W)$, and then we use the result and (7.1) to determine the dimension of the fine homogeneous components of $H_{0}(M \wedge M)$. 
Lemma 7.1. Let $q \vDash n$ with $n \geqslant 5$ such that $k$ of the parts are non-zero.

(i) If at least one part of $q$ is not divisible by 2 , then

$$
\operatorname{dim}\left(H_{0}(W)\right)_{q}=k, \quad \operatorname{dim}\left(H_{1}(W)\right)_{q}=1 .
$$

(ii) If all parts of $q$ are divisible by 2 , then

$$
\operatorname{dim}\left(H_{0}(W)\right)_{q}=k-1, \quad \operatorname{dim}\left(H_{1}(W)\right)_{q}=0 .
$$

Proof. Consider the long exact homology sequence stemming from (7.2). Since $\Delta \otimes P$ is a free $U$-module and hence $H_{k}(\Delta \otimes P)=0$ for all $k \geqslant 1$, this long homology sequence yields isomorphisms

$$
H_{k}(W) \cong H_{k+1}(\Delta \circ \Delta)
$$

for all $k \geqslant 1$, and an exact sequence

$$
0 \rightarrow H_{1}(\Delta \circ \Delta) \rightarrow H_{0}(W) \rightarrow H_{0}(\Delta \otimes P) \rightarrow H_{0}(\Delta \circ \Delta) \rightarrow 0 .
$$

The isomorphism (7.3) with $k=1$ in conjunction with Lemma 6.1 implies the assertions about $H_{1}(W)$. The exact sequence (7.4) implies

$$
\operatorname{dim}\left(H_{0}(W)\right)_{q}=\operatorname{dim}\left(H_{1}(\Delta \circ \Delta)\right)_{q}+\operatorname{dim}\left(H_{0}(\Delta \otimes P)\right)_{q}-\operatorname{dim}\left(H_{0}(\Delta \circ \Delta)\right)_{q} .
$$

By Lemma 4.1, $H_{0}(\Delta \otimes P)$ is a $K$-space with basis consisting of the elements $y_{1} y_{2} \ldots y_{j} \otimes e_{i}$ with $y_{i} \in X, y_{1} \leqslant \cdots \leqslant y_{j}$ and $1 \leqslant i \leqslant r$. There are exactly $k$ such elements of multidegree $q$, and hence $\operatorname{dim}\left(H_{0}(\Delta \otimes P)\right)_{q}=k$. Also, $\operatorname{dim}\left(H_{1}(\Delta \circ \Delta)\right)_{q}$ is given in Lemma 6.1, and $\operatorname{dim}\left(H_{0}(\Delta \circ \Delta)\right)_{q}=1$ by Corollary $5.4(\mathrm{i})$. It remains to put these numbers into the above equation.

At this point, let us state our main result.

Theorem 7.2. Let $G$ be the free centre-by metabelian Lie algebra of rank $r>1$ over a field of characteristic 2 , and let $q \vDash n$ with $n \geqslant 5$ be a composition of $n$ in $r$ parts such that $k$ of the parts are non-zero and $m$ of the parts are equal to 1.

(i) If $m=k$, that is, if $q$ is multilinear, then

$$
\operatorname{dim}\left(G^{\prime \prime}\right)_{q}=\left(\begin{array}{c}
k-1 \\
2
\end{array}\right)
$$

(ii) If at least one of the parts of $q$ is greater than 1 , then

$$
\operatorname{dim}\left(G^{\prime \prime}\right)_{q}= \begin{cases}\left(\begin{array}{c}
k-1 \\
2
\end{array}\right), & \text { if all parts of } q \text { are even } \\
\left(\begin{array}{c}
k \\
2
\end{array}\right)-m, & \text { otherwise. }\end{cases}
$$

Proof. Consider the long exact homology sequence stemming from the short exact sequence (7.1). Its final part is as follows:

$$
\cdots \rightarrow H_{1}(P \wedge P) \rightarrow H_{1}(W) \rightarrow H_{0}(M \wedge M) \rightarrow H_{0}(P \wedge P) \rightarrow H_{0}(W) \rightarrow 0 .
$$

First we prove (i), the case where $q$ is multilinear. In view of Corollary 4.3, then (7.5) yields an exact sequence

$$
0 \rightarrow\left(H_{1}(W)\right)_{q} \rightarrow\left(H_{0}(M \wedge M)\right)_{q} \rightarrow\left(H_{0}(P \wedge P)\right)_{q} \rightarrow\left(H_{0}(W)\right)_{q} \rightarrow 0 .
$$


The exactness of this 4-term exact sequence together with Corollary 5.4(ii) and Lemma 7.1 imply that

$$
\begin{aligned}
\operatorname{dim}\left(H_{0}(M \wedge M)\right)_{q} & =\operatorname{dim}\left(H_{1}(W)\right)_{q}+\operatorname{dim}\left(H_{0}(P \wedge P)\right)_{q}-\operatorname{dim}\left(H_{0}(W)\right)_{q} \\
& =1+\left(\begin{array}{c}
k \\
2
\end{array}\right)-k=\left(\begin{array}{c}
k-1 \\
2
\end{array}\right)
\end{aligned}
$$

as required.

Now we turn to (ii). In this case the exactness of (7.5) implies that

$$
\operatorname{dim}\left(H_{0}(M \wedge M)\right)_{q} \geqslant \operatorname{dim}\left(H_{0}(P \wedge P)\right)_{q}-\operatorname{dim}\left(H_{0}(W)\right)_{q} .
$$

If all parts of $q$ are even, then the right hand side of this inequality is equal to

$$
\operatorname{dim}\left(H_{0}(P \wedge P)\right)_{q}-\operatorname{dim}\left(H_{0}(W)\right)_{q}=\left(\begin{array}{c}
k \\
2
\end{array}\right)-(k-1)=\left(\begin{array}{c}
k-1 \\
2
\end{array}\right),
$$

and when at least one of the parts is odd, then

$$
\operatorname{dim}\left(H_{0}(P \wedge P)\right)_{q}-\operatorname{dim}\left(H_{0}(W)\right)_{q}=\left(\begin{array}{l}
k \\
2
\end{array}\right)+k-m-k=\left(\begin{array}{l}
k \\
2
\end{array}\right)-m .
$$

Hence

$$
\operatorname{dim}\left(H_{0}(M \wedge M)\right)_{q} \geqslant \begin{cases}\left(\begin{array}{c}
k-1 \\
2
\end{array}\right), & \text { if all parts of } q \text { are even; } \\
\left(\begin{array}{c}
k \\
2
\end{array}\right)-m, & \text { otherwise. }\end{cases}
$$

To finish the proof we appeal to Lemma 3.2 of [4] where a generating set of $\left(H_{0}(M \wedge M)\right)_{q}$ is obtained. More precisely, if $q_{i}$ is a part of $q$ such that $q_{i} \geqslant 2$, then the elements

$$
\left[z_{1}, x_{i}\right] \wedge_{*}\left[z_{2}, x_{i}\right]\left(z_{3} z_{4} \ldots z_{n-2}\right),
$$

$z_{1}, z_{2}, \ldots, z_{n-2} \in X$, of multidegree $q$ with $z_{1} \geqslant z_{2}$ and $z_{1}, z_{2} \neq x_{i}$ form a generating set for the fine homogeneous component $\left(H_{0}(M \wedge M)\right)_{q}$. There are $\left(\begin{array}{c}k \\ 2\end{array}\right)-m$ such elements, but if all parts of $q$ are even, then $k-1$ of them are zero, namely the ones with $z_{1}=z_{2}$. Indeed, such elements are of the form $\left[z_{1}, x_{i}\right] \wedge_{*}\left[z_{1}, x_{i}\right] u^{2}$ for some $u \in \mathcal{U}$, and we have, in view of (5.1),

$$
\left[z_{1}, x_{i}\right] \wedge_{*}\left[z_{1}, x_{i}\right] u^{2}=\left[z_{1}, x_{i}\right] u \wedge_{*}\left[z_{1}, x_{i}\right] u=0 .
$$

In either case the number of generators of $\left(H_{0}(M \wedge M)\right)_{q}$ is precisely the number on the right hand side of the inequality (7.6). Hence this number is equal to the dimension of $\left(H_{0}(M \wedge M)\right)_{q}$, as required.

\section{The Free CEntre-By-metabelian Lie Ring}

In this final section we combine our main result with that of [4] to prove a complete structure theorem for the underlying additive group of the free centre-bymetabelian Lie ring, that is, for the case where $K=\mathbb{Z}$. Given $y_{1}, \ldots, y_{n} \in X$ with $n \geqslant 5$, we follow [4] in calling the Lie monomial

$$
\left[\left[y_{1}, y_{2}\right],\left[y_{3}, y_{4}, y_{5}, y_{6}, \ldots, y_{n}\right]\right]
$$


a Kuz'min element if $y_{1}>y_{2}, y_{3}>y_{4}, y_{1} \geqslant y_{3}$ and $y_{4} \leqslant y_{2} \leqslant y_{5} \leqslant \cdots \leqslant y_{n}$, and we call an element of the form

$$
\begin{aligned}
w\left(y_{1}, y_{2}, y_{3}, y_{4} ; y_{5}, \ldots, y_{n}\right)= & {\left[\left[y_{1}, y_{2}\right],\left[y_{3}, y_{4}, y_{5}, \ldots, y_{n}\right]\right] } \\
& +\left[\left[y_{2}, y_{3}\right],\left[y_{1}, y_{4}, y_{5}, \ldots, y_{n}\right]\right] \\
& +\left[\left[y_{3}, y_{1}\right],\left[y_{2}, y_{4}, y_{5}, \ldots, y_{n}\right]\right]
\end{aligned}
$$

a $t$-element.

Theorem 8.1. Let $G$ be the free centre-by-metabelian Lie ring of rank $r>1$ on a free generating set $X=\left\{x_{1}, x_{2}, \ldots, x_{r}\right\}$, let $q=\left(q_{1}, q_{2}, \ldots, q_{r}\right) \vDash n$ be a composition of $n \geqslant 5$, and let $G_{q}^{\prime \prime}$ denote the fine homogeneous component of multidegree $q$ of the second derived ideal $G^{\prime \prime} \subseteq G$.

(i) Suppose that $q=\left(q_{1}, \ldots, q_{r}\right) \vDash n$ is multilinear with $q_{i}=1$ for $i=i_{1}, i_{2}, \ldots, i_{n}$, where $1 \leqslant i_{1}<\cdots<i_{n} \leqslant r$. Then,

(a) if $n$ is odd, $G_{q}^{\prime \prime}$ is generated by the Kuz'min elements of multidegree $q$ and the t-element $w\left(x_{i_{1}}, x_{i_{2}}, x_{i_{3}}, x_{i_{4}} ; x_{i_{5}}, \ldots, x_{i_{n}}\right)$. The former freely generate a free abelian group of rank $\frac{1}{2} n(n-3)$ and the latter generates a cyclic group of order 2 ,

(b) if $n$ is even, then $G_{q}^{\prime \prime}$ is a free abelian group of rank $\left(\begin{array}{c}n-1 \\ 2\end{array}\right)$, and the Kuz'min elements of multidegree $q$ together with the element

$$
\left[\left[x_{i_{3}}, x_{i_{2}}\right],\left[x_{i_{4}}, x_{i_{1}}, x_{i_{5}}, \ldots x_{i_{n}}\right]\right]
$$

form a free generating set for it.

(ii) Suppose that $q=\left(q_{1}, \ldots, q_{r}\right) \vDash n$ is a composition of $n$ with $k$ non-zero parts, such that $q_{i} \geqslant 2$ for some $i$ with $1 \leqslant i \leqslant n$, and $m$ of the parts of $q$ are 1 . Then

(a) if $n$ is odd, $G_{q}^{\prime \prime}$ is a free abelian group of rank $\left(\begin{array}{l}k \\ 2\end{array}\right)-m$, and the elements (8.1) of multidegree $q$ with $y_{2}=y_{4}=x_{i}, y_{1}>y_{3}$ and $y_{1}, y_{3} \neq x_{i}$, form a free generating set for it,

(b) if $n$ is even, then $G_{q}^{\prime \prime}$ is a direct sum of a free abelian group of rank $\left(\begin{array}{c}k-1 \\ 2\end{array}\right)$ that is freely generated by the elements (8.1) of multidegree $q$ with $y_{2}=y_{4}=x_{i}, y_{1}>y_{2}$ and $y_{1}, y_{2} \neq x_{i}$, and an elementary abelian 2group. If at least one of the parts of $q$ is odd, then this 2-group is of rank $k-1-m$, and it is freely generated, as a $\mathbb{Z} / 2 \mathbb{Z}$-module, by the elements (8.1) of multidegree $q$ such that $y_{2}=y_{4}=x_{i}, y_{1}=y_{3} \neq x_{i}$. If all parts of $q$ are even, then the torsion subgroup of $G_{q}^{\prime \prime}$ is zero.

Proof. A substantial part of the theorem is proved in [4, Theorem 7.1]. The only points that were left open in [4] refer to the torsion subgroup $T_{q}$ of $G_{q}^{\prime \prime}$. In part (i.a) of Theorem $7.1 \mathrm{in}$ [4] it is only proved that $T_{q}$ is generated by the $t$-element $w\left(x_{i_{1}}, x_{i_{2}}, x_{i_{3}}, x_{i_{4}} ; x_{i_{5}} \cdots x_{i_{n}}\right)$. The question of whether or not this element is nonzero remained open. Now we can answer this question affirmatively. Indeed, if we reduce $G_{q}^{\prime \prime}$ modulo 2 , then Theorem 7.2 from the previous section tells us that we obtain an elementary abelian 2-group of rank $\left(\begin{array}{c}n-1 \\ 2\end{array}\right)=\frac{1}{2} n(n-3)+1$. By $[4$, Theorem 7.1], $G_{q}^{\prime \prime} / T_{q}$ is a free abelian group of rank $\frac{1}{2} n(n-3)$. Hence the torsion 
subgroup must be non-trivial. In part (ii.b) of Theorem 7.1 in [4] it was proved that if at least one part of $q$ is odd, then the torsion subgroup of $G_{q}^{\prime \prime}$ has exponent 2 and is generated by the elements (8.1) of multidegree $q$ such that $y_{2}=y_{4}=x_{i}$, $y_{1}=y_{3} \neq x_{i}$. Now we can prove that these elements are in fact non-trivial and, moreover, they are linearly independent over $\mathbb{Z} / 2 \mathbb{Z}$. Indeed, by Theorem 7.2 from the previous section, reduction modulo 2 turns $G_{q}^{\prime \prime}$ into an elementary abelian 2group of rank $\left(\begin{array}{c}k \\ 2\end{array}\right)-m$. By [4, Theorem7.1], $G_{q}^{\prime \prime} / T_{q}$ is a free abelian group of rank $\left(\begin{array}{c}k-1 \\ 2\end{array}\right)$. Consequently, the rank of $T_{q}$ as a $\mathbb{Z} / 2 \mathbb{Z}$-module must be $k-1-m$, the number of elements (8.1) such that $y_{2}=y_{4}=x_{i}, y_{1}=y_{3} \neq x_{i}$. Hence these elements are linearly independent over $\mathbb{Z} / 2 \mathbb{Z}$. This completes the proof of theorem.

While part (i) of our theorem agrees with parts 1) and 2) of Theorem 4 in [2], part (ii) contradicts what is claimed in parts 3) and 4) of that theorem. For a more detailed discussion of the discrepancies, see [4].

Acknowledgment. This work was carried out while the second author was visiting the Australian National University. The visit was supported by a Visiting Fellowship from the Mathematical Sciences Institute of the ANU and funds from EPSRC Platform Grant EP/I01912X/1.

\section{REFERENCES}

[1] Yu. A. Bakhturin, Identical relations in Lie algebras, Nauka, Moscow, 1985 (Russian). English translation: VNU Science Press, Utrecht, 1987.

[2] Yu. V. Kuz'min, Free center-by-metabelian groups, Lie algebras and $\mathcal{D}$-groups (Russian), Izv. Akad. Nauk SSSR Ser. Mat. 41 (1977), no. 1, 3-33, 231. English translation: Math. USSR Izvestija 11 (1977), no. 1, 1-30.

[3] Mac Lane, S. Homology, Die Grundlehren der mathematischen Wissenschaften, Bd. 114. Springer-Verlag, Berlin-Göttingen-Heidelberg, 1963.

[4] Nil Mansuroğlu and Ralph Stöhr, Free centre-by-metabelian Lie rings, Q. J. Math. (to appear).

[5] Montgomery, S. Hopf algebras and their actions on rings. CBMS Regional Conference Series in Mathematics, 82. Published for the Conference Board of the Mathematical Sciences, Washington, DC; by the American Mathematical Society, Providence, RI, 1993.

[6] A. L. Šmel'kin, Wreath products of Lie algebras and their application in group theory. (Russian) Trudy Moskov. Mat. Obšč. 29 (1973), 247-260. English Translation: Trans. Mosc. Math. Soc. 29(1973), 239-252 (1976).

[7] Rainer Zerck, On free centre-by-nilpotent-by-abelian groups and Lie rings, Preprint, Akad. Wiss. DDR, Inst. Math. P-MATH-15/91, 29 p. (1991).

Mathematics, Australian National University, Canberra ACT 0200, Australia

E-mail address: kovacs@maths.anu.edu.au

School of Mathematics, University of Manchester, Alan Turing Building, Manchester, M13 9PL, United Kingdom

E-mail address: Ralph.Stohr@manchester.ac.uk 